\title{
Quarter-wavelength nanorod lens based on internal imaging
}

\author{
A. E. Ageyskiy, ${ }^{1}$ S. Yu. Kosulnikov, ${ }^{1}$ S. I. Maslovski, ${ }^{1,2}$ Yu. S. Kivshar,,${ }^{1,3}$ and P. A. Belov ${ }^{1,4}$ \\ ${ }^{1}$ St. Petersburg University of Informational Technologies, Mechanics and Optics, St. Petersburg 197101, Russia \\ ${ }^{2}$ Instituto de Telecomunicações, Universidade de Coimbra, Coimbra, Portugal \\ ${ }^{3}$ Nonlinear Physics Centre, Research School of Physics and Engineering, The Australian National University, Canberra, \\ Australian Capital Territory 0200, Australia \\ ${ }^{4}$ Queen Mary College, University of London, London E1 4NS, United Kingdom
}

(Received 27 September 2011; revised manuscript received 24 November 2011; published 27 January 2012)

\begin{abstract}
We propose a quarter-wavelength metal-backed wire lens for subwavelength imaging based on the internal imaging effect. We analyze the properties of this lens both analytically and numerically and demonstrate that its resolution can be made not worse than $\lambda / 8$.
\end{abstract}

DOI: 10.1103/PhysRevB.85.033105

PACS number(s): 42.30.-d, 78.67.Qa

Numerous super-resolution experiments using various metamaterials have been reported over the last few years. ${ }^{1-10}$ For example, uniaxial lattices of metallic nanorods of resonant length $L=n \lambda / 2$ may operate as subwavelength imaging systems with high resolving power and very low reflectivity. ${ }^{11-16}$ At terahertz $(\mathrm{THz})$ frequencies the conductivity of metallic nanorods is high and, therefore, the dominating modes in a nanorod lattice are the transverse electromagnetic (TEM) waves. ${ }^{17}$ The propagation factor of these waves along the nanorods depends only on the frequency being independent of the field distribution in the transverse plane in a particular mode, i.e., all such modes are transferred through the lens with the same phase delay. Therefore, the modal superposition at the output interface of the lens reconstructs a source near-field picture at the input interface with minimal distortions.

The resonance employed in nanorod lenses is known in optics as the Fabry-Pérot resonance (of the first order when $n=1$ ), and it is responsible for low reflectivity and high transmissivity of these devices. It is also known that the electromagnetic field inside a Fabry-Pérot resonator may experience a resonant growth. Moreover, in uniaxial lattices of metallic nanorods that support propagating modes with large transverse wave numbers, such a growth of the field inside a nanorod lens also happens for plane waves that are evanescent in free space, resulting in the so-called internal imaging phenomenon in wire lenses. ${ }^{18-20}$

In this phenomenon a subwavelength image is formed inside a half-wavelength wire lens at its middle cross section, as shown in Fig. 1(a). At this cross section the magnetic field and the nanorod currents of the TEM modes are maximized, similarly to the magnetic field and the current in a resonant half-wavelength segment of a transmission line open at both ends. It can be shown that the electric field of the TEM modes almost vanishes at the middle cross section; therefore, the image is encoded in the distribution of the nanorod currents.

In this work we study the subwavelength imaging properties of quarter-wavelength nanorod lenses backed with a metal screen. This structure is shown in Fig. 1(b). It is closely related to the internal imaging concept in half-wavelength nanorod lenses. The fact that the electric field practically vanishes at the middle cross section of such lenses allows a metallic screen to be placed there (with nanorods electrically connected to the screen) without disturbing the conditions that are vital for the resonance of the nanorod currents. In practice this means that the nanowires on the other side of the metallic screen may be removed and the lens thickness may be reduced by a factor of 2 without affecting its subwavelength imaging properties. Additionally, placing a conducting screen opens a variety of possibilities for measuring and registering the nanorod currents at the points where the nanorods are attached to it. For example, a charge-coupled device (CCD) matrix could be formed on the ground screen (with any supplementary electronics needed), with nanorods connected to its elements. To register signals at $\mathrm{THz}$ frequencies, various $\mathrm{THz}$ detecting techniques may be used: ${ }^{21}$ direct detection with Schottky diodes, extrinsic semiconductor-based detectors, pyroelectric detectors, etc.

In this Brief Report we concentrate on formulating and solving the electromagnetic part of the problem; therefore, particular semiconductor devices used to detect the currents in the nanorods near the screen are not considered. Instead, the connection of the nanorods to the screen is described with additional boundary conditions (ABCs) developed in Refs. 22-24. These ABCs take into account the nonuniformity in the current and charge distributions in metallic nanowires at the points of interconnections. In the simplest case, when the resistivity (or, more generally, the complex impedance) of the connection is low the corresponding $\mathrm{ABC}$ physically demands vanishing surface charge density at the points where the nanorods meet the metallic screen.

In what follows we consider the quarter-wavelength nanorod lens depicted in Fig. 1(b) which is under the incidence of a $P$-polarized plane electromagnetic wave (propagating or evanescent), as is shown with more detail in Fig. 2. The wave is incident in the $x y$ plane. The nanorod crystal itself is described in the long-wavelength limit with the following uniaxial dielectric permittivity tensor ${ }^{17}$ (the nanorods stand in air):

$$
\varepsilon=\left(\begin{array}{ccc}
1-\frac{k_{p}^{2}}{k^{2}-k_{x}^{2}} & 0 & 0 \\
0 & 1 & 0 \\
0 & 0 & 1
\end{array}\right), \text { where } k_{p}^{2}=\frac{2 \pi / a^{2}}{\ln \frac{a}{2 \pi r}+0.5275} .
$$

The electromagnetic field inside the nanorod crystal may be represented as a superposition of the $P$-polarized TEM and TM waves. The $z$ component of the magnetic field in the whole 
(a)

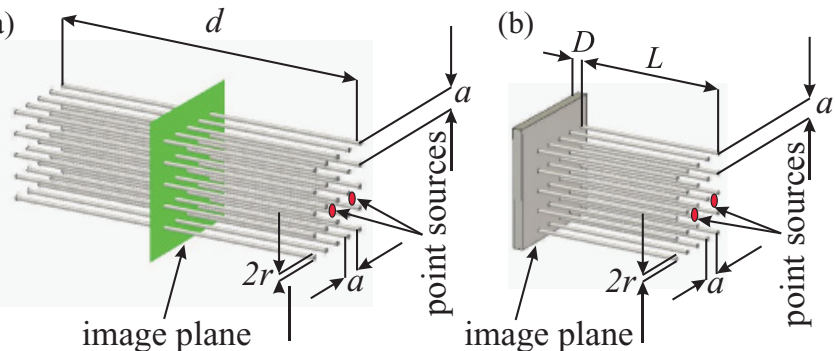

FIG. 1. (Color online) Internal imaging in an array of metallic nanorods with the length (a) $d=\lambda / 2$ and (b) $L=\lambda / 4$. Here, $r$ is the nanorod radius, and $a$ is the lattice period. In (a) the image plane (the green shaded rectangle) is located at the middle of the structure. In the case of panel (b) the nanorods are attached to a metallic screen with thickness $D$ which is much larger than the metal skin depth, and the image plane is located near the screen. The image is encoded in the distribution of the nanorod currents.

structure reads (we normalize this field to the amplitude of the incident field)

$$
\frac{H_{z}(x)}{H_{i}}=\left\{\begin{array}{l}
e^{i k_{x} x}+R e^{-i k_{x} x}, \quad x<0, \\
A_{-}^{\mathrm{TM}} e^{-\gamma_{\mathrm{TM}} x} \\
+A_{+}^{\mathrm{TM}} e^{+\gamma_{\mathrm{TM}} x} \\
+A_{-}^{\mathrm{TEM}} e^{i k x} \\
+A_{+}^{\mathrm{TEM}} e^{-i k x}, \quad 0 \leqslant x \leqslant L,
\end{array}\right.
$$

where $R$ is the unknown reflection coefficient in the air region, $k=\omega / c$ is the free-space wave number, and $k_{x}=i \gamma_{x}$ with $\gamma_{x}=\sqrt{k_{y}^{2}-k^{2}}$ is the $x$ component of the wave vector of the incident wave. $A_{ \pm}^{\mathrm{TM}, \mathrm{TEM}}$ are the unknown amplitudes of the TM and TEM waves in the lens, with the propagation factors $k_{x}^{\mathrm{TM}}=i \gamma_{\mathrm{TM}}$, where $\gamma_{\mathrm{TM}}=\sqrt{k_{p}^{2}+k_{y}^{2}-k^{2}}$, and $k_{x}^{\mathrm{TEM}}=k$, respectively. As is seen, the propagation constant of the TEM mode is independent of $k_{y}$.

In order to find the unknown wave amplitudes, we apply the the boundary conditions at the interfaces $x=0$ and $x=L$. In addition to the classic Maxwellian boundary conditions that require continuity of the tangential components of the fields:

$$
\left[H_{z}\right]=0, \quad\left[\partial H_{z} / \partial x\right]=0,
$$

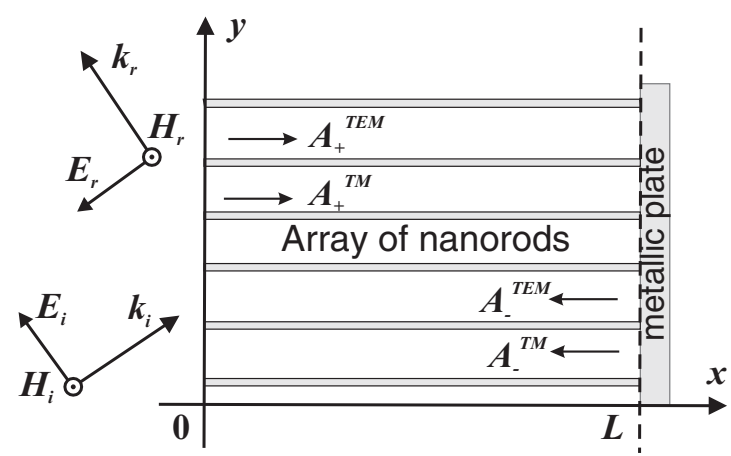

FIG. 2. A plane electromagnetic wave is incident on an array of metallic nanorods connected to a metallic plate. The incident and the reflected waves are TM polarized as indicated by the field vectors $\mathbf{E}_{i}, \mathbf{H}_{i}$ and $\mathbf{E}_{r}, \mathbf{H}_{r}$, and the wave vectors $\mathbf{k}_{i}$ and $\mathbf{k}_{r}$ of the respective waves. Inside the nanorod slab there exist two TM and two TEM waves, the amplitudes of which we denote as $A_{ \pm}^{\mathrm{TM}, \mathrm{TEM}}$.

where $[\cdots]$ denotes the jump of the bracketed quantity across an interface, we notice that when working with a spatially dispersive permittivity of the form (1) one must take into account the ABCs at the ends of the nanorods. ${ }^{24}$

At the plane $x=0$ the $\mathrm{ABC}$ requires that the $x$ component of the conduction current in the nanorods vanishes, $J_{x}=0$, while at the plane $x=L$, where the nanorods are attached to the metal screen, the surface charge on the nanorods must vanish which results in $\partial J_{x} / \partial x=0$. The average density of the conduction current in nanorods can be expressed through the magnetic field:

$$
J_{x}=\frac{i}{k_{y}}\left(\frac{\partial^{2} H_{z}}{\partial x^{2}}+\left(k^{2}-k_{y}^{2}\right) H_{z}\right) .
$$

It is evident that $J_{x}$ as defined by (4) vanishes in the air region. Therefore, taking into account the continuity conditions (3), we may rewrite the two $\mathrm{ABCs}$ in the following form:

$$
\begin{array}{ccc}
\partial^{2} H_{z} / \partial x^{2}=0 & \text { at } & x=0^{+}, \\
\partial^{3} H_{z} / \partial x^{3}=0 & \text { at } & x=L^{-} .
\end{array}
$$

Thus, with the use of (3), (5), and (6) we obtain the following system of linear equations for the unknown reflection coefficient and wave amplitudes:

$$
\left(\begin{array}{ccccc}
-1 & 1 & 1 & 1 & 1 \\
i k_{x} & -\gamma_{\mathrm{TM}} & \gamma_{\mathrm{TM}} & i k & -i k \\
k_{x}^{2} & \gamma_{\mathrm{TM}}^{2} & \gamma_{\mathrm{TM}}^{2} & -k^{2} & -k^{2} \\
0 & -\gamma_{\mathrm{TM}} e^{-\gamma_{\mathrm{TM}} L} & \gamma_{\mathrm{TM}} e^{\gamma_{\mathrm{TM}} L} & i k e^{i k L} & -i k e^{-i k L} \\
0 & -\gamma_{\mathrm{TM}}^{3} e^{-\gamma_{\mathrm{TM}} L} & \gamma_{\mathrm{TM}}^{3} e^{\gamma_{\mathrm{TM}} L} & -i k^{3} e^{i k L} & i k^{3} e^{-i k L}
\end{array}\right)\left(\begin{array}{c}
R \\
A_{-}^{\mathrm{TM}} \\
A_{+}^{\mathrm{TM}} \\
A_{-}^{\mathrm{TEM}} \\
A_{+}^{\mathrm{TEM}}
\end{array}\right)=\left(\begin{array}{c}
1 \\
i k_{x} \\
-k_{x}^{2} \\
0 \\
0
\end{array}\right),
$$

Solving this system, we obtain for the reflection

$$
R=1-\frac{2 i}{k_{x} \Delta}\left[\gamma_{\mathrm{TM}} k_{y}^{2} \tanh \left(\gamma_{\mathrm{TM}} L\right)-k k_{p}^{2} \tan (k L)\right],
$$



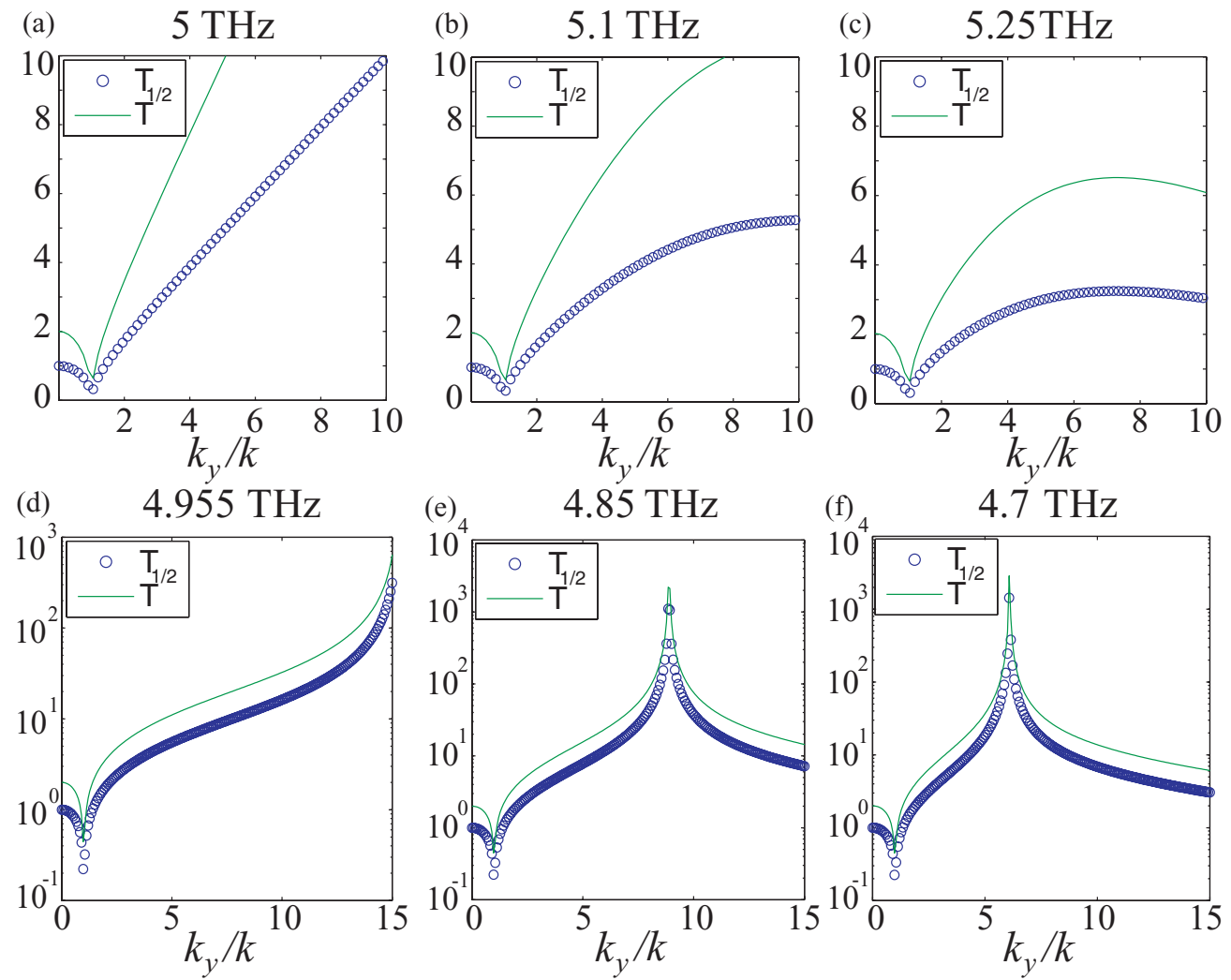

FIG. 3. (Color online) Transmission coefficients $T_{1 / 2}$ and $T$ at operating frequencies above and below the Fabry-Pérot resonance frequency $5 \mathrm{THz}$.

and, for the wave amplitudes inside the lens,

$$
\begin{gathered}
A_{-}^{\mathrm{TM}}=k_{y}^{2} e^{\gamma_{\mathrm{TM}} L} \operatorname{sech}\left(\gamma_{\mathrm{TM}} L\right) / \Delta, \\
A_{+}^{\mathrm{TM}}=k_{y}^{2} e^{-\gamma_{\mathrm{TM}} L} \operatorname{sech}\left(\gamma_{\mathrm{TM}} L\right) / \Delta, \\
A_{-}^{\mathrm{TEM}}=k_{p}^{2} e^{-i k L} \sec (k L) / \Delta, \\
A_{+}^{\mathrm{TEM}}=k_{p}^{2} e^{i k L} \sec (k L) / \Delta,
\end{gathered}
$$

where $\Delta$ is

$$
\begin{aligned}
\Delta= & \left(k_{p}^{2}+k_{y}^{2}\right)\left[1-\frac{k k_{p}^{2}}{\gamma_{x}\left(k_{p}^{2}+k_{y}^{2}\right)} \tan (k L)\right. \\
& \left.+\frac{\gamma_{\mathrm{TM}} k_{y}^{2}}{\gamma_{x}\left(k_{p}^{2}+k_{y}^{2}\right)} \tanh \left(\gamma_{\mathrm{TM}} L\right)\right] .
\end{aligned}
$$

With the solution for the wave amplitudes inside the lens, we may express the magnetic field transmission coefficient from the input plane $x=0$ to the plane $x=L$ where the screen is located as

$$
\begin{aligned}
T & =\left.\frac{H_{z}(x)}{H_{i}}\right|_{x=L} \\
& =A_{-}^{\mathrm{TM}} e^{-\gamma_{\mathrm{TM}} L}+A_{+}^{\mathrm{TM}} e^{\gamma_{\mathrm{TM}} L}+A_{-}^{\mathrm{TEM}} e^{i k L}+A_{+}^{\mathrm{TEM}} e^{-i k L} \\
& =2\left[k_{p}^{2} \sec (k L)+k_{y}^{2} \operatorname{sech}\left(\gamma_{\mathrm{TM}} L\right)\right] / \Delta .
\end{aligned}
$$

For reference, we represent here the transmission coefficient from the input plane to the middle cross section in the half-wavelength lens [see Fig. 1(a); this is the structure in which the internal imaging was first discovered $\left.{ }^{20}\right]$ :

$$
T_{1 / 2}=\left[k_{p}^{2} \sec (k L)-k_{y}^{2} \operatorname{sech}\left(\gamma_{\mathrm{TM}} L\right)\right] / \Delta .
$$

In dense nanorod lenses operating well below the plasma frequency $\omega_{p}=c k_{p}$, the transmission coefficient given by (14) is about twice greater in amplitude when compared to the same given by (15). This follows directly from (14) and (15), as under these conditions $\left|k_{y}^{2} \operatorname{sech}\left(\gamma_{\mathrm{TM}} L\right)\right| \ll\left|k_{p}^{2} \sec (k L)\right|$. Physically, this is due to the reflection of the TEM waves at the metallic screen which doubles the total magnetic field at that point.

It is also interesting to note that the sign of the hyperbolic secant term in (14) is opposite with respect to the sign of the same term in (15). This is related to the fact that the $A B C$ at the metallic screen differs from the $\mathrm{ABC}$ at the open ends of the nanorods.

The plots of the transmission coefficients $T$ and $T_{1 / 2}$ are shown in Figs. 3(a)-3(f). In the case of Fig. 3(a) the lens is tuned exactly at the Fabry-Pérot resonance $(f=5 \mathrm{THz})$. One can see that when $k_{y}>k$ the transfer function $T$ grows practically linearly with $k_{y}$. Physically this may be understood from the known fact that the resonant wire lens reacts to the component of the electric field along the nanowires, $E_{x}$, and this component is such that $E_{x} \propto k_{y} H_{z}$. At frequencies slightly above the Fabry-Pérot resonance the response of the structure is no longer linear with respect to $k_{y}$ at large $k_{y}$, which is seen 


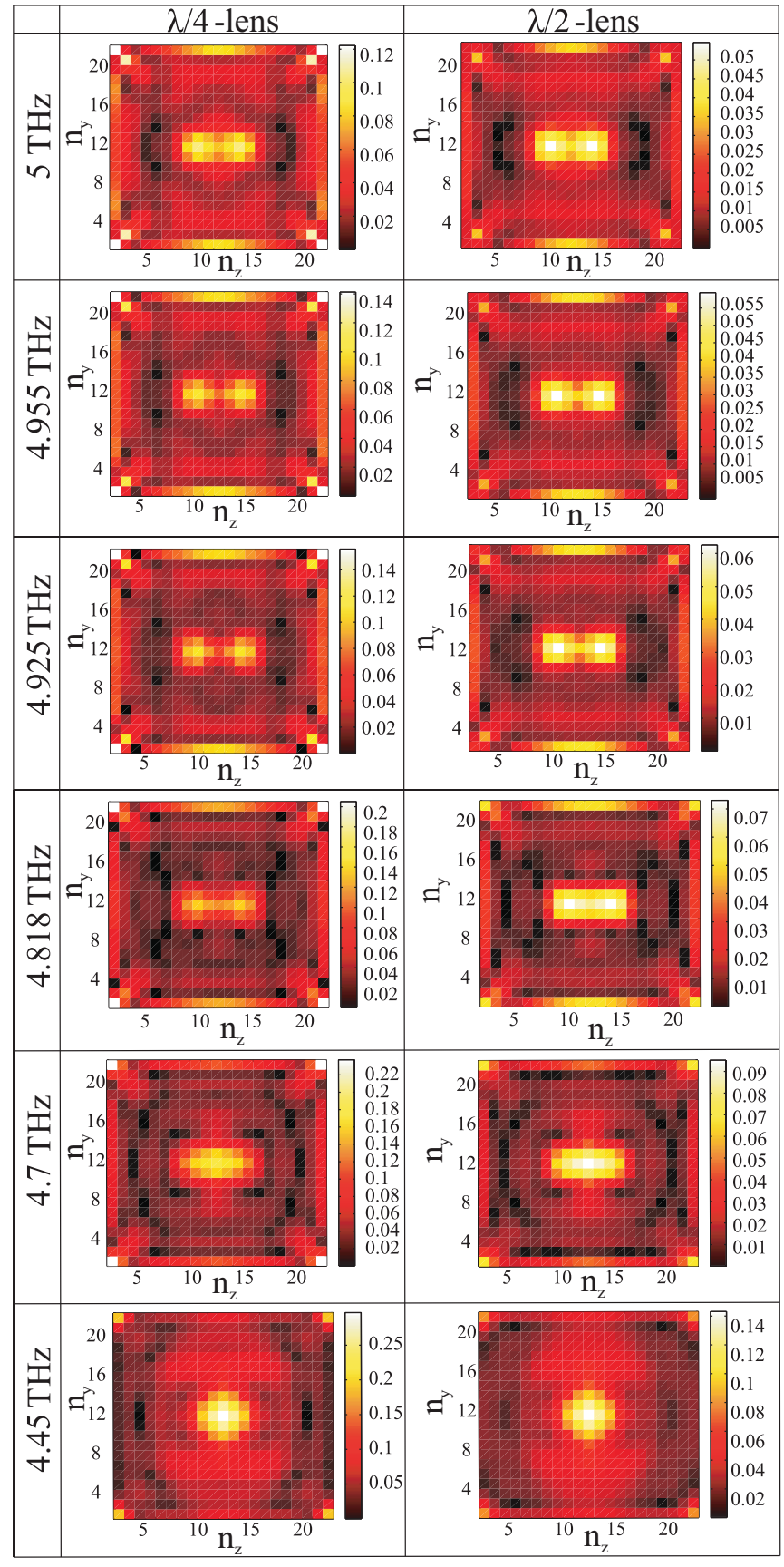

FIG. 4. (Color online) Distributions of the nanorod currents in the image plane. Left: The image of a pair of electric dipoles aligned with the nanorods and placed at $10 \mu \mathrm{m}$ distance from the face of the quarter-wavelength metal-backed lens as encoded in the nanorod currents at the points where the nanorods touch the metal screen. Right: The image of the same sources formed by th half-wavelength lens as encoded in the nanorod currents at the middle cross section of the lens. In both cases the array is formed by $21 \times 21$ metallic nanorods with radius $r=200 \mathrm{~nm}$ and the array period $a=2 \mu \mathrm{m}$. The results are obtained with full-wave simulations in CST MICROWAVE STUDIO. Here, $n_{y}$ and $n_{z}$ are the indices of the nanorods when counted along the $y$ axis and the $z$ axis, respectively. in Figs. 3(b) and 3(c). Nevertheless, the evanescent waves with $k_{y}>k$ are enhanced when tunneled through the lens in all considered cases. Such amplification of the evanescent components may allow for subwavelength imaging of objects that are located at a larger distance from the input interface than with the traditional half-wavelength nanorod lenses operating in canalization mode.

When the frequency is selected slightly below the FabryPérot resonance, guided modes are excited in the structure. Such modes propagating along a grounded slab of nanorods are well known, and they were studied earlier, e.g., in Ref. 25. This situation is observed in Figs. 3(d)-3(f), where the peaks occur at values of $k_{y}$ matching the propagation factors of these guided modes.

Working within a limited range of the transverse wave numbers $k_{y} \in\left(k, k_{\max }\right)$, one may choose an operational frequency at which $k_{\max }<k_{g}$, where $k_{g}$ is the propagation factor of the lowest guided mode (i.e., the location of the first peak). At this frequency the transmission coefficient grows monotonically within the interval $\left(k, k_{\max }\right)$, and it resembles the transfer function of a perfect lens. Thus, the lens resolution may be significantly enhanced when it is operated at this frequency.

To verify this property numerically, we perform a number of numerical simulations in CST MICROWAVE STUDIO. We consider a lens formed by an array of $21 \times 21$ metallic nanorods separated by $a=2 \mu \mathrm{m}$. Two point dipoles oriented along the nanorods and placed at a distance of $10 \mu \mathrm{m}$ from the first interface of the lens are used as the near-field source. The results of the simulations are presented in Fig. 4, where we plot the distributions of the nanorod currents in the quarter-wavelength lens at the plane where the nanorods are attached to the screen (the image plane) (Fig. 4, left), and also the same distributions in the half-wavelength lens at its middle cross section (Fig. 4, right), at different frequencies within the range $4.45 \leqslant f \leqslant 5 \mathrm{THz}$. As follows from these results, the resolution of both types of lens is not worse than $\lambda / 8$, while the quarter-wavelength lens appears more promising in practice due to its smaller thickness and better access to the nanorod currents in which the image is encoded.

In conclusion, we have proposed and studied a subwavelength imaging system formed by an array of quarterwavelength nanorods attached to a metallic screen. The lens operation is based on the internal imaging principle. When the nanorods are attached to a metallic screen, the resonant length of the nanorods may be twice smaller than in the case of free-standing nanorods; thus, one may realize a quarter-wavelength nanorod lens in which the subwavelength imaging occurs at the screen plane where the image is encoded in the nanorod currents. Various methods may be used to detect these currents at $\mathrm{THz}$ frequencies; however, in the present work we have concentrated only on the electromagnetic properties of the lens. We have developed an analytical theory of such metal-backed lenses and verified this theory by numerical simulations. Both the theory and simulations predict a mode of operation of the lens in which the incident evanescent plane waves are resonantly enhanced within the lens, which opens a possibility for realizing subwavelength imaging devices of an improved resolution when compared with the other techniques; the resolution is not worse than $\lambda / 8$ and mostly determined by the period of the nanorod array. 
${ }^{1}$ Z. Liu, H. Lee, Y. Xiong, C. Sun, and X. Zhang, Science 315, 1686 (2007).

${ }^{2}$ S. Kawata, A. Ono, and P. Verma, Nature Photon. 2, 438 (2008).

${ }^{3}$ K. Aydin, I. Bulu, and E. Ozbay, Appl. Phys. Lett. 90, 254102 (2007).

${ }^{4}$ B. D. F. Casse, W. T. Lu, Y. J. Huang, E. Gultepe, L. Menon, and S. Sridhar, Appl. Phys. Lett. 96, 023114 (2010).

${ }^{5}$ F. Lemoult, G. Lerosey, J. de Rosny, and M. Fink, Phys. Rev. Lett. 104, 203901 (2010).

${ }^{6}$ M. J. Freire, L. Jelinek, R. Marques, and M. Lapine, J. Magn. Reson. 203, 81 (2010).

${ }^{7}$ J. Jung, F. J. Garcia-Vidal, L. Martin-Moreno, and J. B. Pendry, Phys. Rev. B 79, 153407 (2009).

${ }^{8}$ A. Ono, J. I. Kato, and S. Kawata, Phys. Rev. Lett. 95, 267407 (2005).

${ }^{9}$ A. Salandrino and N. Engheta, Phys. Rev. B 74, 075103 (2006).

${ }^{10}$ L. Shi and L. Gao, Phys. Rev. B 77, 195121 (2008).

${ }^{11}$ P. A. Belov and M. G. Silveirinha, Phys. Rev. E 73, 056607 (2006).

${ }^{12}$ P. A. Belov, Y. Zhao, S. Tse, P. Ikonen, M. G. Silveirinha, C. R. Simovski, S. Tretyakov, Y. Hao, and C. Parini, Phys. Rev. B 77, 193108 (2008).

${ }^{13}$ M. G. Silveirinha, P. Belov, and C. R. Simovskiy, Opt. Lett. 33, 1726 (2008)
${ }^{14}$ P. Belov, G. Palikaras, Y. Zhao, A. Rahman, C. Simovski, Y. Hao, and C. Parini, Appl. Phys. Lett. 97, 191905 (2010).

${ }^{15}$ A. Rahman, P. Belov, Y. Hao, and C. Parini, Opt. Lett. 35, 142 (2010).

${ }^{16}$ Y. Zhao, G. Palikaras, P. Belov, R. Dubrovka, C. Simovski, Y. Hao, and C. Parini, New J. Phys. 12, 103045 (2010).

${ }^{17}$ P. A. Belov, R. Marques, S. I. Maslovski, I. S. Nefedov, M. Silveirinha, C. R. Simovski, and S. A. Tretyakov, Phys. Rev. B 67, 113103 (2003).

${ }^{18}$ Y. Zhao, P. Belov, and Y. Hao, J. Opt. A: Pure Appl. Opt. 11, 075101 (2009).

${ }^{19}$ P. Belov, Y. Zhao, Y. Hao, and C. Parini, Opt. Lett. 34, 527 (2009).

${ }^{20}$ A. E. Ageyskiy, S. Y. Kosulnikov, and P. A. Belov, Opt. Spectrosc. 110, 572 (2011).

${ }^{21}$ A. Rogalski and F. Sizov, Opto-Electron. Rev. 19, 346 (2011).

${ }^{22}$ M. G. Silveirinha, IEEE Trans. Antennas Propag. 54, 1766 (2006).

${ }^{23}$ M. Silveirinha, C. A. Fernandes, and J. Costa, New J. Phys. 10, 053011 (2008).

${ }^{24}$ S. I. Maslovski, T. A. Morgado, M. G. Silveirinha, C. S. R. Kaipa, and A. B. Yakovlev, New J. Phys. 12, 113047 (2010).

${ }^{25}$ M. G. Silveirinha, C. A. Fernandes, and J. R. Costa, IEEE Trans. Antennas Propag. 56, 405 (2008). 